\title{
Right weak Engel conditions on linear groups
}

\section{B. A. F. Wehrfritz ${ }^{1}$}

Received: 5 June 2019 / Accepted: 28 October 2019 / Published online: 12 December 2019

(c) The Author(s) 2019

\begin{abstract}
If $\mathbf{X}$ is a group-class, a group $\mathrm{G}$ is right $\mathbf{X}$-Engel if for all $\mathrm{g}$ in $\mathrm{G}$ there exists an $\mathbf{X}$-subgroup $E$ of $G$ such that for all $x$ in $G$ there is a positive integer $m(x)$ with $\left[g,{ }_{n} x\right] \in E$ for all $n \geq m(x)$. Let $G$ be a linear group. Special cases of our main theorem are the following. If $\mathbf{X}$ is the class of all Chernikov groups, or all finite groups, or all locally finite groups, then $\mathrm{G}$ is right $\mathbf{X}$-Engel if and only if $\mathrm{G}$ has a normal $\mathbf{X}$-subgroup modulo which $\mathrm{G}$ is hypercentral. The same conclusion holds if $\mathrm{G}$ has positive characteristic and $\mathbf{X}$ is one of the following classes; all polycyclic-by-finite groups, all groups of finite Prüfer rank, all minimax groups, all groups with finite Hirsch number, all soluble-by-finite groups with finite abelian total rank. In general the characteristic zero case is more complex.
\end{abstract}

Keywords Engel conditions $\cdot$ Rank conditions $\cdot$ Linear group $\cdot$ Matrix group

\section{Mathematics Subject Classification 20F45 · 20E34 $\cdot$ 20H20}

In Ref. [4] Khukhro and Shumyatsky introduced a weakened Engel condition for groups, which they named almost Engel (below we give full definitions of this and other relevant Engel conditions). It is effectively a weakening of the notion of a left Engel group. In their later paper [5] they consider a right version of their condition that they call right almost Engel, after of course renaming almost Engel left almost Engel.

Between these two papers Shumyatsky [9] characterised (left) almost Engel linear groups. The referee of the first version of this paper has referred me to Ref. [10] of which I was unaware. In this Shumyatsky characterises right almost Engel linear groups. Meanwhile I broadened the discussion by introducing a wide range of analogous conditions for linear groups in Refs. [14-16], called there $\mathbf{X}$-Engel groups for $\mathbf{X}$ one of a number of classes of groups. We now wish to rename these left $\mathbf{X}$-Engel groups, since here we discuss analogies of Khukhro and Shumyatsky's right almost Engel condition.

Thus let $\mathbf{X}$ be a class, either the class $\mathbf{F}_{\text {set }}$ of finite sets or a class of groups in the usual sense (e.g. [7], vol. 1, page 1). A group $G$ is left $\mathbf{X}$-Engel if for all $\mathrm{g}$ in $\mathrm{G}$ there exists an $\mathbf{X}$ subset/subgroup $E$ of $G$ such that for all $x$ in $G$ there is a positive integer $m(x)$ with $\left[x,{ }_{n} g\right.$ ] $\in \mathrm{E}$ for all $\mathrm{n} \geq \mathrm{m}(\mathrm{x})$. Here $\mathrm{E}$ is called a sink for $\mathrm{g}$ in $\mathrm{G}$. A group $\mathrm{G}$ is right $\mathbf{X}$-Engel if for all $\mathrm{g}$ in $\mathrm{G}$ there exists an $\mathbf{X}$ subset/subgroup $\mathrm{E}$ of $\mathrm{G}$ such that for all $\mathrm{x}$ in $\mathrm{G}$ there is a positive

$\otimes$ B. A. F. Wehrfritz

b.a.f.wehrfritz@qmul.ac.uk

1 Queen Mary University of London, Mile End Road, London E1 4NS, England 
integer $\mathrm{m}(\mathrm{x})$ with $\left[\mathrm{g},{ }_{\mathrm{n}} \mathrm{x}\right] \in \mathrm{E}$ for all $\mathrm{n} \geq \mathrm{m}(\mathrm{x})$. Here we call $\mathrm{E}$ a knis for $\mathrm{g}$ in $\mathrm{G}$. (Khukhro and Shumyatsky use the terms left sink and right sink for our sink and knis, but I have grown tired of seeing endless streams of left's and right's.) Thus left/right $\mathbf{F}_{\text {set }}$-Engel is just the left/right almost Engel of Refs. [4, 5] and $\mathbf{F}_{\text {set }}$ is just a symbolic way of writing 'almost' so that we can present all cases in a common format.

As well as $\mathbf{F}_{\text {set }}$ we wish to consider quite a number of group classes, although in practice they can be considered in a much smaller number of batches. However it is very convenient to have a short notation for each; see Ref. [6] for definitions of the terms below. Thus:

F denotes the class of all finite groups,

LF denotes the class of all locally finite groups,

Ch denotes the class of all Chernikov groups,

$\mathbf{P}$ denotes the class of all polycyclic groups so,

PF denotes the class of all polycyclic-by-finite groups,

$\mathbf{X}_{\mathrm{fr}}$ denotes the class of all groups with finite (Prüfer) rank,

$\mathbf{X}_{\mathrm{ftr}}$ denotes the class of all soluble-by-finite groups with finite abelian total rank,

$\mathbf{X}_{\mathrm{mm}}$ denotes the class of all minimax groups and

$\mathbf{X}_{\mathrm{fh}}$ denotes the class of all groups with finite Hirsch number (= torsion-free rank).

Lemma 1 of Ref. [16] gives the interrelations between these classes for linear groups; they depend upon the ground-field characteristic.

Now in linear groups right Engel elements are always left Engel, e.g. [12] 8.15, so one might expect right $\mathbf{X}$-Engel linear groups to tend to be left $\mathbf{X}$-Engel. This is indeed the case. The $\mathbf{F}_{\text {set }}$ case of Part i) of the theorem below is effectively Shumyatsky's main result in Ref. [10].

Theorem Let $\mathrm{G}$ be a subgroup of $\mathrm{GL}(\mathrm{n}, \mathrm{F})$, where $\mathrm{n}$ is a positive integer and $\mathrm{F}$ is a field of characteristic $\mathrm{p} \geq 0$.

i) If $\mathbf{X}$ is one of $\mathbf{F}_{\mathrm{set}}, \mathbf{F}, \mathbf{C h}$ or $\mathrm{LF}$, then the following are equivalent.

a) $\mathrm{G}$ is right $\mathbf{X}$-Engel.

b) $\mathrm{G}$ is $\mathbf{X}$-by-hypercentral.

c) $\mathrm{G}$ is left $\mathbf{X}$-Engel.

ii) If $\mathrm{p}>0$ and $\mathbf{X}$ is one of $\mathbf{P F}, \mathbf{X}_{\mathrm{fr}}, \mathbf{X}_{\mathrm{ftr}}, \mathbf{X}_{\mathrm{mm}}$ or $\mathbf{X}_{\mathrm{fh}}$ then again $\left.\mathrm{a}\right)$, b) and c) above are equivalent.

iii) If $\mathrm{p}=0$ and $\mathrm{G}$ is right $\mathbf{P F}$-Engel, then $\mathrm{G}$ is left $\mathbf{P F}-$ Engel.

iv) Let $\mathrm{p}=0$ and let $\mathbf{X}$ be one of $\mathbf{X}_{\mathrm{fr}}, \mathbf{X}_{\mathrm{ftr}}, \mathbf{X}_{\mathrm{mm}}$ or $\mathbf{X}_{\mathrm{fh}}$. If $\mathrm{G}$ is (right $\mathbf{X}$-Engel)-by-finite, then $\mathrm{G}$ is (left $\mathbf{X}$-Engel)-by-finite. Suppose $\mathrm{G}$ is triangularizable or (Zariski) connected. If $\mathrm{G}$ is right $\mathbf{X}$-Engel, then $\mathrm{G}$ is left $\mathbf{X}$-Engel.

In Refs. [14-16] we derive precise descriptions of the class of left $\mathbf{X}$-Engel linear groups for the various choices of $\mathbf{X}$, so the proof of the theorem basically comes down to proving that right $\mathbf{X}$-Engel linear groups satisfy these descriptions. The simplest of these descriptions is given by b) in i) and ii) above. In Refs. $[14,16]$ we prove that b) and c) are equivalent under the hypotheses of i) and ii) and trivially b) always implies a). Thus for i) and ii) it remains only to check that a) implies b). For $p=0$, apart from the simple cases covered in $i$ ), the descriptions are much more complex, but in general terms our approach to the proofs of iii) and iv) is similar.

In connection with Part iii) of the theorem, Example 2 in Ref. [15] is a nilpotent-by-finite linear group of degree 3 over the rational numbers, that is left PF-Engel but not PF-by- 
hypercentral. However it is also right PF-Engel, as a very simple direct check shows. This example is not connected, but we give a connected example at the end of this paper.

Lemma 1 (See [5] Lemma 2.5) Let $\mathrm{G}$ be a metabelian group. If $\mathrm{n}$ is a positive integer and $\mathrm{x}$ and $\mathrm{g}$ are elements of $\mathrm{G}$, then $[\mathrm{x}, \mathrm{n}+2 \mathrm{~g}]=\left[\mathrm{g}^{-1},{ }_{\mathrm{n}} \mathrm{g}^{[\mathrm{g}, \mathrm{x}] \mathrm{g}}\right]$. If $\mathrm{E}$ is a knis for $\mathrm{g}^{-1}$ in $\mathrm{G}$, then $\mathrm{E}$ is also a sink for $\mathrm{g}$ in $\mathrm{G}$. In particular for any class $\mathbf{X}$ the group $\mathrm{G}$ is left $\mathbf{X}$-Engel whenever $\mathrm{G}$ is right $\mathbf{X}$-Engel.

Lemma 2 Let $\mathrm{G}$ be a group, $\mathrm{M}$ a subgroup of $\mathrm{G}, \mathrm{N}$ a normal subgroup of $\mathrm{G}$ and $\mathrm{L}=\mathrm{K}$ [A, the split extension of the abelian subgroup $\mathrm{A}$ of $\mathrm{G}$ by the abelian subgroup $\mathrm{K}$ of $\mathrm{N}_{\mathrm{G}}(\mathrm{A}) / \mathrm{C}_{\mathrm{G}}(\mathrm{A})$. Suppose $\mathrm{G}$ is right (resp. left) $\mathbf{X}$-Engel for some group class $\mathbf{X}$. Then:

a) $\mathrm{M}$ is right (resp. left) $\mathrm{SX}$-Engel.

b) $\mathrm{G} / \mathrm{N}$ is right (resp. left) QX-Engel.

c) L is left $\mathrm{SX}$-Engel in both cases.

Moreover if $\mathrm{G}$ is right (resp. left) $\mathbf{F}_{\mathrm{set}}$-Engel, then so are $\mathrm{M}$ and $\mathrm{G} / \mathrm{N}$ and $\mathrm{L}$ is left $\mathbf{F}_{\mathrm{set}^{-}}$ Engel in both cases.

Here $\mathrm{S}$ denotes the subgroup operator and $\mathrm{Q}$ the quotient-group operator.

Proof The claims for $\mathrm{M}$ and $\mathrm{G} / \mathrm{N}$ are obvious. Let $\mathrm{ha} \in \mathrm{L}$, where $\mathrm{h}=\mathrm{gC}_{\mathrm{G}}(\mathrm{A}) \in \mathrm{K}$ and $\mathrm{a} \in \mathrm{A}$ and $g \in G$. If $G$ is left $\mathbf{X}$-Engel, then $\langle\mathrm{g}\rangle \mathrm{A}$ is left $\mathbf{S X}$-Engel. If $\mathrm{G}$ is right $\mathbf{X}$-Engel, then $\langle\mathrm{g}\rangle \mathrm{A}$ is right $\mathbf{S X}$-Engel and hence left $\mathbf{S X}$-Engel by Lemma 1. Either way there is an SX-subgroup $\mathrm{E}$ of $\mathrm{A}$ such that for all $\mathrm{d}$ in $\mathrm{A}$ and all large enough $\mathrm{n}$ ( $\mathrm{n}$ depending on $\mathrm{d}$ of course) we have $\left[\mathrm{d},{ }_{\mathrm{n}} \mathrm{g}\right] \in \mathrm{E}$. If $\mathrm{kb} \in \mathrm{L}$ (where $\mathrm{k} \in \mathrm{K}$ and $\mathrm{b} \in \mathrm{A}$ ), since $\mathrm{K}$ is abelian, $\mathrm{d}=[\mathrm{kb}$, ha $] \in \mathrm{A}$. Then $\left[\mathrm{kb},{ }_{\mathrm{n}+1} \mathrm{ha}\right]=[\mathrm{d}, \mathrm{ng}]$ for all $\mathrm{n} \geq 1$ and consequently $\left[\mathrm{kb},{ }_{\mathrm{n}+1} \mathrm{ha}\right] \in \mathrm{E}$ for all large enough $\mathrm{n}$. Therefore L is left SX-Engel. The claims for $\mathbf{F}_{\text {set }}$ follow similarly.

Suppose A is a subgroup of the multiplicative group $\mathrm{F}^{*}$ of the field $\mathrm{F}$ and $\mathrm{B}$ is a subgroup of the additive group $\mathrm{F}^{+}$of $\mathrm{F}$ such that $\mathrm{AB}=\mathrm{B}$. Consider the split extension $\mathrm{C}=\mathrm{A}[\mathrm{B}$ of $\mathrm{B}$ by $\mathrm{A}$. If $\mathrm{C}$ is right $\mathbf{X}$-Engel for some class $\mathbf{X}$, then by Lemma 1 the group $\mathrm{C}$ is also left $\mathbf{X}$-Engel. Results for the left case in Refs. [14-16] easily yield the following lemma. Notice that as a consequence of this lemma if $\mathbf{X}$ is any of the nine classes that it covers, then $\mathrm{C}$ is left $\mathbf{X}$-Engel if and only if $\mathbf{C}$ is right $\mathbf{X}$-Engel.

Lemma 3 Suppose $\mathrm{C}$ is left $\mathbf{X}$-Engel.

a) If $\mathbf{X}$ is $\mathbf{F}_{\mathrm{set}}, \mathbf{F}$ or $\mathbf{C h}$, then $\mathrm{A}=\langle 1\rangle$ or $\mathrm{B}$ is finite.

b) If charF $>0$ and $\mathbf{X}$ is $\mathbf{X}_{\mathrm{fr}}, \mathbf{X}_{\mathrm{ftr}}, \mathbf{X}_{\mathrm{mm}}$ or $\mathbf{P F}$ then again $\mathrm{A}=\langle 1\rangle$ or $\mathrm{B}$ is finite.

c) If charF $=0, \mathrm{~A} \neq\langle 1\rangle, \mathrm{B} \neq\langle 0\rangle$ and $\mathbf{X}$ is $\mathbf{P F}$ or $\mathbf{X}_{\mathrm{mm}}$, then $\mathrm{A}$ is finitely generated and $\mathrm{B}$ is minimax. Moreover if $\mathbf{X}=\mathbf{P F}$, then $\mathrm{B}$ contains a normal, finitely generated subgroup $\mathrm{E}$ of $\mathrm{C}$ with $\mathrm{B} / \mathrm{E}$ Chernikov and $\mathrm{C}$-hypercentral.

d) If charF $=0$ and $\mathbf{X}$ is $\mathbf{X}_{\mathrm{fr}}, \mathbf{X}_{\mathrm{ftr}}$ or $\mathbf{X}_{\mathrm{fh}}$, then $\mathrm{A}=\langle 1\rangle$ or $\mathrm{B}$ has finite rank.

e) If charF $=0$ and $\mathbf{X}$ is $\mathbf{F}_{\mathrm{set}}, \mathbf{F}, \mathbf{C h}$ or $\mathrm{LF}$, then $\mathrm{A}=\langle 1\rangle$ or $\mathrm{B}=\langle 0\rangle$.

Note that in b) if $\mathbf{X}$ is $\mathbf{X}_{\mathrm{fh}}$ or LF clearly there is no restriction on A and B. Also in c) if $\mathrm{A}=\langle 2\rangle \leq \mathbf{Q}^{*}$ and $\mathrm{B}=\mathbb{Z}[1 / 2]$, then clearly $\mathrm{C}$ is right $\mathbf{X}_{\mathrm{mm}}$-Engel, but there is no finitely generated subgroup $\mathrm{E}$ of $\mathrm{B}$ that is normal in $\mathrm{C}$ with $\mathrm{B} / \mathrm{E}$ Chernikov.

Proof a) Let $\mathrm{a} \in \mathrm{A}$ and $\mathrm{b} \in \mathrm{B}$. If $\mathbf{X}=\mathbf{F}_{\text {set }}$ then $\mathrm{b}(\mathrm{a}-1)^{\mathrm{m}}=\mathrm{b}(\mathrm{a}-1)^{\mathrm{n}}$ for some $1 \leq \mathrm{m}<\mathrm{n}$. If $a \neq 1$ then $b=b(a-1)^{n-m}=b(a-1)^{r(n-m)}$ for any $r \geq 1$. Consequently $B$ lies in any sink of a and hence $B$ is finite. The same applies if $\mathbf{X}=\mathbf{F}$. If $\mathbf{X}=\mathbf{C h}$ then since sinks can always be chosen in $\mathrm{B} \geq \mathrm{C}^{\prime}$ and Chernikov subgroups of $\mathrm{B}$ are always finite, so $\mathrm{B}$ is also left $\mathbf{F}$-Engel and the previous case applies. 
b) Here B is elementary abelian, so $\mathbf{X}_{\mathrm{fr}}$-subgroups of B are finite. Hence in these cases $\mathrm{C}$ is also left $\mathbf{F}$-Engel and a) applies.

c) If $\mathbf{X}=\mathbf{P F}$ the claims follow from Proposition 1 of Ref. [15]. If $\mathbf{X}=\mathbf{X}_{\mathrm{mm}}$ they follow from Lemma 7 of Ref. [16].

d) If $\mathbf{X}=\mathbf{X}_{\mathrm{fr}}$ or $\mathbf{X}_{\mathrm{ftr}}$ the claim follows from Ref. [16] Lemma 7 again. If $\mathbf{X}=\mathbf{X}_{\mathrm{fh}}$ it follows from Ref. [16] Lemmas 1 and 7.

e) Here $\langle 0\rangle$ is the only finite subgroup of $\mathrm{B}$. Thus a) yields that $\mathrm{A}=\langle 1\rangle$ or $\mathrm{B}=\langle 0\rangle$ in each case except possibly $\mathbf{X}=\mathrm{LF}$. But sinks can always be chosen in B, so this case is covered too.

Say that a group $\mathrm{G}$ is weakly $\mathbf{X}$-Engel if for all $\mathrm{x}$ and $\mathrm{y}$ in $\mathrm{G}$ there is an integer $\mathrm{m}=$ $\mathrm{m}(\mathrm{x}, \mathrm{y}) \geq 1$ and $\mathrm{E} \in \mathbf{X}$, a subset of $\mathrm{G}$, such that $[\mathrm{x}, \mathrm{ny}] \in \mathrm{E}$ for all $\mathrm{n} \geq \mathrm{m}$. Clearly both right and left $\mathbf{X}$-Engel groups are weakly $\mathbf{X}$-Engel.

Lemma 4 Let $\mathrm{G}$ be a weakly $\mathbf{X}$-Engel subgroup of $\mathrm{GL}(\mathrm{n}, \mathrm{F}), \mathrm{n}$ a positive integer and $\mathrm{F}$ some field, where $\mathbf{X}=\mathbf{F}_{\text {set }}$ or $\mathbf{X}$ is a group class with $\mathbf{X}=\mathbf{S X}$ (meaning $\mathbf{X}$ is subgroup-closed) that does not contain the free group of rank 2. Then $\mathrm{G}$ is soluble-by-finite if charF $=0$ and is soluble-by-(locally finite) otherwise.

Proof We copy the proof of Ref. [16] Lemma 2. If $\mathrm{H} \leq \mathrm{G}$ is free of rank 2, say on $\{\mathrm{x}, \mathrm{y}\}$, then the commutators $[\mathrm{x}, \mathrm{n} y]$ for $\mathrm{n} \geq 1$ are all distinct. Also $\langle[\mathrm{x}, \mathrm{n} \mathrm{y}]: \mathrm{n} \geq \mathrm{m}\rangle$ is not cyclic for all $\mathrm{m} \geq 1$. Thus $\mathrm{H}$ cannot be weakly $\mathbf{X}$-Engel for any $\mathbf{X}$ as in the lemma. Lemma 4 now follows at once from Tits' Theorem ([12] 10.17).

Lemma 5 Let $\mathrm{G}$ be either a left or a right $\mathbf{X}$-Engel subgroup of $\mathrm{GL}(\mathrm{n}, \mathrm{F})$, where $\mathrm{n}$ is a positive integer and $\mathrm{F}$ a field of positive characteristic and either $\mathbf{X}=\mathbf{F}_{\mathrm{set}}$ or $\mathbf{X}$ is a group class satisfying $\mathbf{X}=\mathrm{SX}=\mathrm{QX} \subseteq \mathrm{X}_{\mathrm{fr}}$. Then

$\mathrm{G}$ is soluble-by-finite.

$\mathbf{X}=\mathrm{QX}$ means that homomorphic images of $\mathbf{X}$-groups lie in $\mathbf{X}$.

Proof Suppose G is not soluble-by-finite. By Lemma 4 (and [12] 6.4, 5.9 and 5.11) we may assume that the soluble radical of $\mathrm{G}$ is $\langle 1\rangle$ and that $\mathrm{G}$ is locally finite. Then by Ref. [8] 5.1.5 we may assume that $\mathrm{G}$ is infinite simple and hence (see [11]) of Lie type over some infinite locally finite field $k_{0}$ of characteristic p. Then by Ref. [1] 6.3.1 we may assume that $G$ is $\operatorname{PSL}(2, \mathrm{k})$ for some infinite subfield $\mathrm{k}$ of $\mathrm{k}_{\mathrm{o}}$ and hence $\mathrm{G}$ contains a copy of $\mathrm{J}=\left(\mathrm{k}^{*}\left[\mathrm{k}^{+}\right) /\langle-1\rangle\right.$, where $\mathrm{k}^{+}$is the additive group of $\mathrm{k}, \mathrm{k}^{*}$ is the multiplicative group of $\mathrm{k}, \mathrm{k}^{*}$ acts on $\mathrm{k}^{+}$via squares and $\langle-1\rangle \leq \mathrm{k}^{*}$.

Clearly $\mathbf{J}$ is metabelian, so by Lemmas 1 and 2 the group $\mathbf{J}$ is left $\mathbf{X}$-Engel. Hence each element of $\mathbf{J}$ has an $\mathbf{X}$-sink in $\mathrm{k}^{+}$and $\mathrm{k}^{+}$is elementary abelian. But $\mathbf{X} \subseteq \mathbf{X}_{\text {fr }}$ or $\mathbf{X}=\mathbf{F}_{\text {set }}$. Therefore $\mathrm{J}$ and the split extension $\mathrm{k}^{*}\left[\mathrm{k}^{+}\right.$are left $\mathbf{F}$-Engel. Consequently $\mathrm{k}$ is finite by Lemma 3a), which it is not. Lemma 5 now follows.

Lemma 6 Let $\mathrm{A} \leq \mathrm{T}$ be normal subgroups of a group $\mathrm{G}$ with $[\mathrm{A}, \mathrm{T}]=\langle 1\rangle$ and $\mathrm{G} / \mathrm{T}$ finite. Let $\mathbf{X}$ be a group class such that for each $\mathrm{g}$ in $\mathrm{G}$ there is a knis $\mathrm{E}(\mathrm{g}) \in \mathbf{X}$ for $\mathrm{g}$ in $\mathrm{G}$. If

a) $\mathbf{X}=\mathrm{SX}$ and whenever $\mathrm{U}, \mathrm{V} \leq \mathrm{W}$, where $\mathrm{W}$ is an abelian group and $\mathrm{U}$ and $\mathrm{V}$ lie in $\mathbf{X}$, then $\mathrm{UV} \in \mathbf{X}$, or

b) $\mathbf{X}=\mathrm{S} \mathbf{X}=\mathrm{Q} \mathbf{X}=\mathrm{D}_{\mathrm{O}} \mathbf{X}$ or

c) $\mathbf{X}=\mathbf{X}_{\mathrm{ftr}}$, 
then there is a normal $\mathbf{X}$-subgroup $\mathrm{B} \leq \mathrm{A}$ of $\mathrm{G}$ with $\mathrm{A} / \mathrm{B} \leq \zeta_{\omega}(\mathrm{G} / \mathrm{B})$. If also $\mathrm{A}=\mathrm{T}$, then $\mathrm{G}$ is XF-by-hypercentral.

Note that the following classes satisfy b); $\mathbf{X}_{\mathrm{fr}}, \mathbf{X}_{\mathrm{mm}} . \mathbf{X}_{\mathrm{fh}}, \mathbf{C h}, \mathbf{P}, \mathbf{P F}, \mathbf{F}$ and LF. ( $\mathrm{D}_{\mathrm{O}} \mathbf{X}=$ $\mathbf{X}$ means that the direct product of two $\mathbf{X}$-groups lies in $\mathbf{X}$.)

Proof Let $\mathrm{g} \in \mathrm{G}$. Then $\mathrm{H}=\langle\mathrm{g}\rangle \mathrm{A}$ is metabelian and $\mathrm{H} \cap \mathrm{E}\left(\mathrm{g}^{-1}\right)$ is a knis for $\mathrm{g}^{-1}$ in $\mathrm{H}$. Then $\mathrm{H} \cap \mathrm{E}\left(\mathrm{g}^{-1}\right)$ is a sink for $\mathrm{g}$ in $\mathrm{H}$ by Lemma 1. But $\mathrm{A}$ is normal in $\mathrm{H}$, so $\mathrm{A} \cap \mathrm{E}\left(\mathrm{g}^{-1}\right)$ is a sink for $\mathrm{g}$ in $\mathrm{A}$. The conclusions of Lemma 6 now follow from Lemma 5 of Ref. [16].

For any group $\mathrm{G}$ denote its upper central series by $\left\{\zeta_{\mathrm{s}}(\mathrm{G}): 0 \leq \mathrm{s} \leq \sigma\right\}$, s and $\sigma$ ordinals and its hypercentre by $\zeta(\mathrm{G})=\cup_{\mathrm{s}} \zeta_{\mathrm{s}}(\mathrm{G})$.

Lemma 7 Let $\mathrm{A} \leq \mathrm{T}$ be normal subgroups of the right $\mathbf{P F}$-Engel group $\mathrm{G}$ with $\mathrm{G} / \mathrm{T}$ finite.

a) If $\mathrm{A}$ is abelian and Chernikov with $\mathrm{A} \leq \zeta(\mathrm{T})$, then there exists $\mathrm{r} \geq 1$ such that $\mathrm{A}(\mathrm{r})=\{\mathrm{a} \in$ $\left.\mathrm{A}: \mathrm{a}^{\mathrm{r}}=1\right\}$ is finite, normal in $\mathrm{G}$ and satisfies $\mathrm{A} / \mathrm{A}(\mathrm{r}) \leq \zeta_{\omega}(\mathrm{G} / \mathrm{A}(\mathrm{r}))$.

b) If $[\mathrm{A}, \mathrm{T}]=\langle 1\rangle$ then there exists a finitely generated subgroup $\mathrm{B} \leq \mathrm{A}$ normal in $\mathrm{G}$ such that $\mathrm{A} / \mathrm{B} \leq \zeta_{\omega}(\mathrm{G} / \mathrm{B})$.

Proof Let $X=\left\{g_{1}, g_{2}, \ldots, g_{t}\right\}$ be a transversal of $T$ to $G$. Then each $g_{i}^{-1}$ has a knis $E_{i}$ in $G$ with $E_{i} \in \mathbf{P F}$. In particular each $B_{i}=A \cap E_{i}$ is finitely generated and abelian. Now each $G_{i}$ $=\left\langle g_{i}\right\rangle$ A is metabelian, so by Lemma 1 each $G_{i} \cap E_{i}$ is a sink for $g_{i}$ in $G_{i}$.

a) Here each $B_{i}$ is finite, so $\cup_{i} B_{i} \subseteq A(r)$ for some $r \geq 1$ and clearly $A(r)$ is finite and normal in $\mathrm{G}$. Now $\mathrm{A}$ is Chernikov and $\mathrm{A} \leq \zeta(\mathrm{T})$, so there is a normal series

$$
\mathrm{A}(\mathrm{r})=\mathrm{A}_{0} \leq \mathrm{A}_{1} \leq \cdots \leq \mathrm{A}_{\omega}=\mathrm{A}
$$

of $\mathrm{G}$, where each $\mathrm{A}_{\mathrm{i}+1} / \mathrm{A}_{\mathrm{i}}$ is finite and T-central. Let $\mathrm{a} \in \mathrm{A}_{\mathrm{i}+1}$ and $\mathrm{g} \in \mathrm{G}$. Then $\mathrm{g}=\mathrm{hg}_{\mathrm{i}}$ for some $i$ and some $h \in T$. Hence $\left[a,{ }_{n} g\right] \in\left[a,{ }_{n} g_{i}\right] A_{i}$ for all $n \geq 1$ and $\left[a,{ }_{n} g_{i}\right] \in A \cap E_{i}$ $\leq A(r)$ for all large enough $n$. Therefore $A_{i+1} / A_{i} \leq \zeta\left(G / A_{i}\right)$, e.g. by Ref. [12] 8.1. But $A_{i+1} / A_{i}$ is finite; consequently $A / A(r) \leq \zeta_{\omega}(G / A(r))$.

b) Set $\mathrm{B}=\left\langle\mathrm{B}_{\mathrm{i}}^{\mathrm{g}}: \mathrm{g} \in \mathrm{G}, 1 \leq \mathrm{i} \leq \mathrm{t}\right\rangle=\left\langle\mathrm{B}_{\mathrm{i}}^{\mathrm{g}}: \mathrm{g} \in \mathrm{X}, 1 \leq \mathrm{i} \leq \mathrm{t}\right\rangle$. Then $\mathrm{B} \leq \mathrm{A}$ is finitely generated and normal in $\mathrm{G}$. Also $\mathrm{A} / \mathrm{B}$ consists of right Engel elements of $\mathrm{G} / \mathrm{B}$ and Ref. [12] 8.1 again yields that $\mathrm{A} / \mathrm{B} \leq \zeta_{\omega}(\mathrm{G} / \mathrm{B})$.

Lemma 8 Let $\mathrm{G}$ be a right $\mathbf{F}_{\mathrm{set}}$-Engel, nilpotent-by-finite group. Then $\mathrm{G}$ is finite-byhypercentral.

This is a right-handed version of Ref. [14] Lemma 2.1.

Proof Note first that a group $G$ is finite-by-hypercentral if and only if $G / \zeta(G)$ is finite, see Refs. [2, 3].

Let $\mathrm{N}$ be a nilpotent normal subgroup of the right $\mathbf{F}_{\text {set }}$-Engel group $\mathrm{G}$ of finite index and set $A=\zeta_{1}(\mathrm{~N})$. By induction on the class of $\mathrm{N}$ we may assume that $\mathrm{H} / \mathrm{A}=\zeta(\mathrm{G} / \mathrm{A})$ has finite index in G/A. If $\mathrm{g} \in \mathrm{G}$, then $\langle\mathrm{g}\rangle \mathrm{A}$ is metabelian. Therefore $\langle\mathrm{g}\rangle \mathrm{A}$ is also left $\mathbf{F}_{\text {set }}$-Engel by Lemma 1 and hence by Ref. [14] Lemma $2.1\langle\mathrm{~g}\rangle \mathrm{A}$ has a finite normal subgroup $\mathrm{E}_{\mathrm{g}}$ such that $\langle\mathrm{g}\rangle \mathrm{A} / \mathrm{E}_{\mathrm{g}}$ is hypercentral. Clearly $\langle\mathrm{g}\rangle \mathrm{A} / \mathrm{A}$ is abelian, so we may choose $\mathrm{E}_{\mathrm{g}}$ in A. Note that for each $a$ in $A$ there exists $m=m(g, a)$ with $[a, m g] \in E_{g}$.

Let $X$ be a (finite) transversal of $N$ to $G$ and consider $g \in G$ and $a \in A$. Then $g=h x$ for some $\mathrm{h} \in \mathrm{N}$ and $\mathrm{x} \in \mathrm{X}$ and $\left[\mathrm{a},{ }_{\mathrm{n}} \mathrm{g}\right]=\left[\mathrm{a},{ }_{\mathrm{n}} \mathrm{x}\right]$ for any $\mathrm{n} \geq 1$. Set

$$
\mathrm{E}=\left\langle\left(\mathrm{E}_{\mathrm{x}}\right)^{\mathrm{y}}: \mathrm{x}, \mathrm{y} \in \mathrm{X}\right\rangle \leq \mathrm{A} \text {. }
$$


Since each $E_{x}$ is central in $N$ and $A$ is abelian, so $E$ is a finite normal subgroup of $G$. Also $\mathrm{A} / \mathrm{E}$ consists of right Engel elements of $\mathrm{G} / \mathrm{E}$ and $\left(\mathrm{G}: \mathrm{C}_{\mathrm{G}}(\mathrm{A})\right)$ is finite. Hence $\mathrm{A} / \mathrm{E} \leq \zeta(\mathrm{G} / \mathrm{E})$ by Ref. [12] 8.1 and therefore $\zeta(\mathrm{G} / \mathrm{E}) \geq \mathrm{H} / \mathrm{E}$, which is of finite index in $\mathrm{G} / \mathrm{E}$. Consequently there is a finite normal subgroup $\mathrm{L} / \mathrm{E}$ of $\mathrm{G} / \mathrm{E}$ with $\mathrm{G} / \mathrm{L}$ hypercentral. Trivially $\mathrm{L}$ is finite, which completes the proof of Lemma 8.

\section{Proof of Parts i) and ii) of the Theorem}

Let $\mathrm{G}$ be a right $\mathbf{X}$-Engel subgroup of $\mathrm{GL}(\mathrm{n}, \mathrm{F})$, where $\mathrm{n}$ is a positive integer and $\mathrm{F}$ is a field of characteristic $p \geq 0$. To prove parts i) and ii) of the Theorem, it suffices to prove, under suitable conditions on $\mathbf{X}$, that $\mathrm{G}$ is $\mathbf{X}$-by-hypercentral, see comments after the statement of the Theorem.

a) Suppose $\mathbf{X}$ is $\mathbf{F}_{\text {set }}$ or $\mathbf{F}$. Then $\mathrm{G}$ is finite-by-hypercentral.

By Lemmas 4 and 5 the group $\mathrm{G}$ is soluble-by-finite, so $\mathrm{T}=\mathrm{G}^{\mathrm{o}}$, the connected component of $\mathrm{G}$ containing 1, is triangularizable (Lie-Kolchin Theorem). Let $\mathrm{U}=\mathrm{u}(\mathrm{T})$ denote its unipotent radical, so $\mathrm{T}^{\prime} \leq \mathrm{U}$. There is a normal series

$$
\langle 1\rangle=\mathrm{U}_{0} \leq \mathrm{U}_{1} \leq \cdots \leq \mathrm{U}_{\mathrm{t}}=\mathrm{U}, \quad \text { where } \mathrm{t} \leq \mathrm{n}(\mathrm{n}-1) / 2,
$$

of $\mathrm{T}$ such that for each $\mathrm{i}$ we have $\left[\mathrm{U}_{\mathrm{i}}, \mathrm{U}\right] \leq \mathrm{U}_{\mathrm{i}-1}$ and either $\mathrm{U}_{\mathrm{i}} / \mathrm{U}_{\mathrm{i}-1}$ is finite or $\left[\mathrm{U}_{\mathrm{i}}, \mathrm{T}\right] \leq \mathrm{U}_{\mathrm{i}-1}$ (use Lemmas 2 and 3a). A simple induction on t (e.g. using Ref. [7] 4.25 and 4.21) yields that $\mathrm{T}$ is finite-by-nilpotent and hence nilpotent-by-finite. Therefore $\mathrm{G}$ is finiteby-hypercentral by Lemma 8 .

b) Suppose $\mathbf{X}=\mathbf{C h}$. Then $\mathrm{G} / \zeta(\mathrm{G})$ is Chernikov and $\mathrm{G}$ is Chernikov-by-nilpotent.

By Lemmas 4 and 5 the subgroup $\mathrm{T}=\mathrm{G}^{\mathrm{o}}$ is triangularizable. If $\mathrm{g} \in \mathrm{T}$ there is a knis $\mathrm{E}$ for $\mathrm{g}$ in $\mathrm{T}$ contained in $\mathrm{T}^{\prime} \leq \mathrm{u}(\mathrm{T})$ with $\mathrm{E}$ Chernikov. But $\mathrm{u}(\mathrm{T})$ is torsion-free or of finite exponent, Therefore $\mathrm{E}$ is finite and $\mathrm{T}$ is right $\mathbf{F}$-Engel. By a) above $\mathrm{T}$ is finiteby-hypercentral and hence is hypercentral-by-finite. A triangular locally nilpotent linear group is nilpotent (e.g. by Ref. [12] 4.13). Hence $\mathrm{G}$ has a nilpotent normal subgroup $\mathrm{N}$ of finite index. We may choose $\mathrm{N}$ closed in $\mathrm{G}$ ([12] 5.11 and 5.9) and then $\mathrm{A}=\zeta_{1}(\mathrm{~N})$ is also closed in G. Consequently G/A is right $\mathbf{C h}$-Engel as well as isomorphic to a linear group. By induction on the class of $\mathrm{N}$ we may assume that $\mathrm{G} / \mathrm{A}$ modulo $\zeta(\mathrm{G} / \mathrm{A})$ is Chernikov. By Lemma $6 b$ ) there exists a Chernikov subgroup B of A, normal in $\mathrm{G}$ and with $A / B \leq \zeta(G / B)$. Hence $G / B$ modulo $\zeta(G / B)$ is Chernikov, so by Ref. [13] Theorem B there is a Chernikov normal subgroup L/B of G/B with G/L hypercentral. Clearly L is Chernikov. The claim b) now follows from Ref. [14] Proposition 1.3.

c) Suppose $\mathrm{p}>0$ and $\mathbf{X} \subseteq \mathbf{X}_{\mathrm{fr}}$ with $\mathbf{X}=\mathrm{S} \mathbf{X}=\mathrm{Q} \mathbf{X}=\mathrm{D}_{\mathrm{o}} \mathbf{X}=\mathbf{X F}$; e.g. $\mathbf{X}=\mathbf{X}_{\mathrm{fr}}, \mathbf{X}_{\mathrm{mm}}$ or $\mathbf{P F}$. Then $\mathrm{G}$ is $\mathbf{X}$-by-hypercentral.

By Lemma 5 the group $G$ is soluble-by-finite, $T=G^{o}$ is triangularizable and $T^{\prime} \leq u(T)$ has finite exponent. But $\mathbf{X}_{\mathrm{fr}}$-groups of finite exponent are finite. Therefore $\mathrm{T}$ is right F-Engel and hence by a) is finite-by-hypercentral, hypercentral-by-finite and nilpotentby-finite. Thus $\mathrm{G}$ is nilpotent-by-finite and therefore $\mathrm{G}$ has a nilpotent closed connected normal subgroup $\mathrm{N}$ of finite index. By Remark 3.3 of Ref. [14] it suffices to consider just two cases, $\mathrm{N}$ unipotent and $\mathrm{N}$ a d-group. If $\mathrm{N}$ is unipotent every $\mathbf{X}$-subgroup of $\mathrm{G}$ is finite, so by a) the group $\mathrm{G}$ is finite-by-hypercentral. Clearly $\mathbf{F} \subseteq \mathbf{X}$. If $\mathrm{N}$ is a d-group, then $\mathrm{N}$ is abelian and the index $\left(\mathrm{G}: \mathrm{C}_{\mathrm{G}}(\mathrm{N})\right)$ is finite (dividing $\mathrm{n}$ ! in fact, see [12] 1.12). Then $\mathrm{G}$ is $\mathbf{X}$-by-hypercentral by Lemma 6 b). The proof of c) is complete.

d) Suppose $\mathrm{p}>0$ and $\mathbf{X}=\mathbf{X}_{\mathrm{ftr}}$. Then $\mathrm{G}$ is $\mathbf{X}$-by-hypercentral.

By c) there is a normal $\mathbf{X}_{\mathrm{fr}}$-subgroup $\mathrm{X}$ of $\mathrm{G}$ with $\mathrm{G} / \mathrm{X}$ hypercentral. Then $\mathrm{X}^{\mathrm{o}}$ is an abelian normal subgroup of $G$ of finite index in $X$, see [16] Lemma 1. By Ref. [16] Lemma 4 there is a normal subgroup $\mathrm{Y}$ of $\mathrm{G}$ of finite index in $\mathrm{X}^{\mathrm{o}}$ with $\left(\mathrm{G}: \mathrm{C}_{\mathrm{G}}(\mathrm{Y})\right.$ ) dividing $\mathrm{n}$ !. By 
Lemma 6c) there is a normal $\mathbf{X}_{\mathrm{ftr}}$-subgroup $\mathrm{Z}$ of $\mathrm{G}$ with $\mathrm{Z} \leq \mathrm{Y}$ and $\mathrm{Y} / \mathrm{Z} \leq \zeta(\mathrm{G} / \mathrm{Z})$. Now $\mathrm{X} / \mathrm{Y}$ is finite and $\mathrm{G} / \mathrm{X}$ is hypercentral. Hence $(\mathrm{G} / \mathrm{Y}: \zeta(\mathrm{G} / \mathrm{Y}))$ is finite by Ref. [2]. But then $(\mathrm{G} / \mathrm{Z}: \zeta(\mathrm{G} / \mathrm{Z}))$ is finite, so by Ref. [3] there exists $\mathrm{L} / \mathrm{Z}$ finite and normal in G/Z such that $\mathrm{G} / \mathrm{L}$ is hypercentral. Clearly $\mathrm{L} \in \mathbf{X}_{\mathrm{ftr}}$.

e) Suppose $\mathrm{p}>0$ and $\mathbf{X}=\mathbf{X}_{\mathrm{fh}}$. Then $\mathrm{G}$ is $\mathbf{X}$-by hypercentral.

By Lemma 4 there exists a closed triangularizable normal subgroup $\mathrm{N}$ of $\mathrm{G}$ with $\mathrm{G} / \mathrm{N}$ locally finite. Then $\mathrm{N}^{\prime} \leq \mathrm{u}(\mathrm{N})=\mathrm{O}_{\mathrm{p}}(\mathrm{N})$, which is also closed in $\mathrm{G}$, so $\mathrm{H}=\mathrm{G} / \mathrm{O}_{\mathrm{p}}(\mathrm{N})$ is isomorphic to a linear group of characteristic p, see Ref. [12] 6.4. The image $M$ of $N$ in $\mathrm{H}$ is abelian with $\mathrm{O}_{\mathrm{p}}(\mathrm{M})=\langle 1\rangle$ and there exists a power $\mathrm{q}$ of $\mathrm{p}$, in fact $\mathrm{p}$ to the power of the degree of the chosen faithful representation of $\mathrm{H}$, such that $\mathrm{M}^{\mathrm{q}}$ is diagonalizable. Then ( $\mathrm{H}: \mathrm{C}_{\mathrm{H}}\left(\mathrm{M}^{\mathrm{q}}\right)$ ) is finite ([12] 1.12 again) and Lemma $6 \mathrm{~b}$ ) yields that $\mathrm{M}^{\mathrm{q}}$ contains

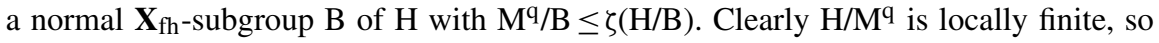
H/B contains, by Ref. [13] Theorem D, a locally finite normal subgroup L/B with H/L hypercentral. Defining $\mathrm{D} \leq \mathrm{G}$ by $\mathrm{D} / \mathrm{O}_{\mathrm{p}}(\mathrm{N})=\mathrm{L}$, we have that $\mathrm{D}$ is a normal $\mathbf{X}_{\mathrm{fh}}$-subgroup of $\mathrm{G}$ with $\mathrm{G} / \mathrm{D}$ hypercentral.

f) Suppose $\mathbf{X}=\mathrm{LF}$. Then $\mathrm{G}$ is $\mathbf{X}$-by-hypercentral.

If $p>0$, we repeat the proof of e); specifically Lemma $6 b$ ) here yields $B$ as above but with B a LF-subgroup. If $p=0$ we may simply repeat the proof of Ref. [16] Theorem 4, noting that in the first paragraph of that proof, if $\mathrm{R}$ is right LF-Engel, then $\mathrm{Q}$ consists of right Engel elements of $\mathrm{R}$ (just as it did when R was left LF-Engel). Then proceed as in Ref. [16], but now with Lemma 4 above yielding that $\mathrm{G}$ is soluble-by-finite.

Note that we have now completed the proof of Parts i) and ii) of the Theorem, with a), b) and f) yielding Part i) and c), d) and e) yielding Part ii). There is an alternative approach to this that makes more use of the topology. A step in the proofs of Sections a), b) and c) and hence indirectly in the proofs of Sections d), e) and f) of i) and ii) is to show that the groups $\mathrm{G}$ in question are nilpotent-by-finite. Applying Lemmas 4 and 5 as in the proofs of a), b) and $\mathrm{c}$ ), the Lie-Kolchin Theorem then yields that $\mathrm{T}=\mathrm{G}^{\mathrm{o}}$ is triangularizable and, of course, connected. In Lemma 3 if $\mathrm{B} \neq\langle 0\rangle$ then $\mathrm{A}$ acts faithfully on $\mathrm{B}$, so if $\mathrm{A}$ is also connected, then $A$ is $\langle 1\rangle$ or infinite. Thus in Parts a), b) and e) of Lemma 3, if $A$ is connected then $A=$ $\langle 1\rangle$. From this we can deduce, under the hypotheses of Sections a), b) or c) in the proof of Parts i) and ii) the theorem, that $u(T) \leq \zeta(T)$ and hence that $T$ is nilpotent. Consequently $G$ is nilpotent-by-finite and then the proofs of Sections a), b), c) and hence of d), e) and f) can be completed as before.

Proposition 1 Let $\mathrm{G}$ be a right $\mathbf{P F}$-Engel subgroup of $\mathrm{GL}(\mathrm{n}, \mathrm{F})$ where $\mathrm{n} \geq 1$ and charF $=0$. Then $\mathrm{G}$ has a normal series

$$
\langle 1\rangle=\mathrm{T}_{0} \leq \mathrm{T}_{1} \leq \mathrm{T}_{2} \leq \cdots \leq \mathrm{T}_{\mathrm{s}}=\mathrm{T} \leq \mathrm{G}
$$

of finite length, where $\mathrm{G} / \mathrm{T}$ is finite and each $\mathrm{T}_{\mathrm{i}} / \mathrm{T}_{\mathrm{i}-1}$ is polycyclic-by-finite, or $\mathrm{G}$ hypercentral with $\left[\mathrm{T}_{\mathrm{i}}, \mathrm{T}\right] \leq \mathrm{T}_{\mathrm{i}-1}$, or $\mathrm{G}$-hypercentral, abelian and Chernikov.

Proof By Lemma 4 the group $\mathrm{G}$ is soluble-by-finite, so $\mathrm{T}=\mathrm{G}^{\mathrm{o}}$ is triangularizable. Set $\mathrm{U}=$ $\mathrm{u}(\mathrm{T})$. Exactly as in the proof of the Theorem of Ref. [15], but using Lemma 3c) instead of Ref. [15] Proposition 1, there is a normal series

$$
\langle 1\rangle=\mathrm{U}_{0} \leq \mathrm{V}_{1} \leq \mathrm{U}_{1} \leq \mathrm{V}_{2} \leq \mathrm{U}_{2} \leq \cdots \leq \mathrm{V}_{\mathrm{t}} \leq \mathrm{U}_{\mathrm{t}}=\mathrm{U}
$$

of $\mathrm{T}$ such that for each $\mathrm{i}$ we have the following: $\left[\mathrm{U}_{\mathrm{i}}, \mathrm{U}\right] \leq \mathrm{U}_{\mathrm{i}-1}, \mathrm{~T} / \mathrm{C}_{\mathrm{T}}\left(\mathrm{U}_{\mathrm{i}} / \mathrm{U}_{\mathrm{i}-1}\right)$ is finitely generated, $\mathrm{V}_{\mathrm{i}} / \mathrm{U}_{\mathrm{i}-1}$ is finitely generated abelian, $\mathrm{U}_{\mathrm{i}} / \mathrm{V}_{\mathrm{i}}$ is $\mathrm{T}$-hypercentral and either $\left[\mathrm{U}_{\mathrm{i}} \mathrm{T}\right] \leq \mathrm{U}_{\mathrm{i}-1}$ 
or $\mathrm{U}_{\mathrm{i}} / \mathrm{V}_{\mathrm{i}}$ is abelian and Chernikov. Replacing each term in this series by its normal closure in $\mathrm{G}$ yields a series with these same properties but also with each term normal in $\mathrm{G}$.

For each i Lemma 7 yields a subgroup $\mathrm{W}_{\mathrm{i}}$ normal in $\mathrm{G}$ with $\mathrm{V}_{\mathrm{i}} \leq \mathrm{W}_{\mathrm{i}} \leq \mathrm{U}_{\mathrm{i}}$, with $\mathrm{W}_{\mathrm{i}} / \mathrm{V}_{\mathrm{i}}$ finitely generated abelian and with $\mathrm{U}_{\mathrm{i}} / \mathrm{W}_{\mathrm{i}} \mathrm{G}$-hypercentral. Finally Lemma $7 \mathrm{~b}$ ) applied to $\mathrm{G} / \mathrm{U}$ yields a normal subgroup $\mathrm{B}$ of $\mathrm{G}$ with $\mathrm{U} \leq \mathrm{B} \leq \mathrm{T}, \mathrm{B} / \mathrm{U}$ finitely generated abelian and $\mathrm{T} / \mathrm{B} \leq \zeta(\mathrm{G} / \mathrm{B})$. Clearly $[\mathrm{T}, \mathrm{T}] \leq \mathrm{B}$. Thus the series

$$
\langle 1\rangle=\mathrm{U}_{0} \leq \mathrm{W}_{1} \leq \mathrm{U}_{1} \leq \mathrm{W}_{2} \leq \mathrm{U}_{2} \leq \cdots \leq \mathrm{W}_{\mathrm{t}} \leq \mathrm{U}_{\mathrm{t}}=\mathrm{U} \leq \mathrm{B} \leq \mathrm{T} \leq \mathrm{G}
$$

has the required properties.

Proposition 2 Let $\mathrm{T}$ be a right $\mathbf{X}$-Engel subgroup of $\operatorname{Tr}(\mathrm{n}, \mathrm{F})$, where $\mathrm{n} \geq 1$, charF $=0$ and $\mathbf{X}$ is $\mathbf{X}_{\mathrm{fr}}, \mathbf{X}_{\mathrm{ftr}}$ or $\mathbf{X}_{\mathrm{mm}}$. Then $\mathrm{T}$ has a normal series

$$
\langle 1\rangle=\mathrm{U}_{0} \leq \mathrm{U}_{1} \leq \mathrm{U}_{2} \leq \cdots \leq \mathrm{U}_{\mathrm{t}}=\mathrm{U} \leq \mathrm{T}
$$

of finite length such that $\mathrm{T} / \mathrm{U}$ is abelian, $\mathrm{U}$ is torsion-free and for each $\mathrm{i} \geq 1$ we have $\left[\mathrm{U}_{\mathrm{i}}, \mathrm{U}\right] \leq \mathrm{U}_{\mathrm{i}-1}$ and either $\mathrm{U}_{\mathrm{i}} / \mathrm{U}_{\mathrm{i}-1} \in \mathbf{X}$ or $\left[\mathrm{U}_{\mathrm{i}}, \mathrm{T}\right] \leq \mathrm{U}_{\mathrm{i}-1}$.

Proof Let $\mathrm{U}=\mathrm{u}(\mathrm{T})$. Then $\mathrm{U}$ is torsion-free and $\mathrm{T} / \mathrm{U}$ is abelian. The existence of suitable $\mathrm{U}_{1}$, $\mathrm{U}_{2}, \ldots, \mathrm{U}_{\mathrm{t}}$ follow from Lemma 3, Parts c) and d) with each $\mathrm{U}_{\mathrm{i}} / \mathrm{U}_{\mathrm{i}-1}$ torsion-free. The latter ensures that if $\mathrm{U}_{\mathrm{i}} / \mathrm{U}_{\mathrm{i}-1}$ lies in $\mathbf{X}_{\mathrm{fr}}$, then it lies in $\mathbf{X}_{\mathrm{ftr}}$.

\section{Proof of Parts iii) and iv) of the Theorem}

In Part iii) if G is right $\mathbf{X}$-Engel, then by Proposition 1, the group $\mathrm{G}$ satisfies the hypotheses of Ref. [15] Proposition 2. Hence G is also left $\mathbf{X}$-Engel.

Now consider Part iv). Suppose $\mathbf{X}$ is $\mathbf{X}_{\mathrm{fr}}, \mathbf{X}_{\mathrm{ftr}}$ or $\mathbf{X}_{\mathrm{mm}}$. If $\mathrm{G}$ is right $\mathbf{X}$-Engel and connected, then $G$ is triangularizable by Lemma 4 . If $G$ is triangularizable, then $G$ is left $\mathbf{X}$-Engel by Proposition 2 and [16] Proposition 3. If $\mathrm{G}$ has a right $\mathbf{X}$-Engel normal subgroup $\mathrm{H}$ of finite index, then $\mathrm{H}^{\mathrm{o}}$ is right $\mathbf{X}$-Engel and hence left $\mathbf{X}$-Engel. Clearly $\mathrm{H}^{\mathrm{o}}$ is normal of finite index in G. Finally since charF $=0$, each $\mathbf{X}_{\mathrm{fh}}$-subgroup of GL(n, F) lies in $\mathbf{X}_{\mathrm{fr}}$ by Ref. [16] Lemma 1 , so $\mathrm{G}$ is right (resp. left) $\mathbf{X}_{\mathrm{fh}}$-Engel if and only if it is right (resp. left) $\mathbf{X}_{\mathrm{fr}}$-Engel. Part iv) of the Theorem follows.

Example There exists a connected triangular linear group of degree 6 over the rational numbers that is both left and right PF-Engel but is not PF-by-hypercentral.

Let $\mathrm{a}=\left(\mathrm{a}_{\mathrm{ij}}\right) \in \mathrm{GL}(2, \mathbb{Z})$, where $\mathrm{a}_{11}=7, \mathrm{a}_{12}=2, \mathrm{a}_{21}=10$ and $\mathrm{a}_{22}=3$. Then $\operatorname{det}(\mathrm{a})=$ $1, \mathrm{a} \cong 1 \bmod 2$ and the eigenvalues of a are $\lambda=5-\sqrt{ } 24$ and $\lambda^{-1}=5+\sqrt{ } 24$. In particular $0<\lambda<1<\lambda^{-1}$ and $a$ is an element of $\operatorname{GL}(2, \mathbb{Z})$ of infinite order. Further $\langle a\rangle$ is connected; to see this it suffices to prove that its conjugate $\left\langle\mathrm{a}^{\prime}\right\rangle=\left\langle\operatorname{diag}\left(\lambda, \lambda^{-1}\right)\right\rangle$ is connected. This follows since $\left\langle\mathrm{a}^{\prime}\right\rangle$ and $\langle\lambda\rangle \leq \mathbb{R}^{*}$ are group and topologically isomorphic via the obvious maps.

Set $\mathrm{J}=\mathbb{Z}[1 / 2], A=\mathrm{J}^{2 \times 2}$, the ring of 2 by 2 matrices over $\mathrm{J}$, and $\mathrm{B}=\mathbb{Z}^{2 \times 2}<\mathrm{A}$. Then $\mathrm{A}$ is a right $\langle\mathrm{a}\rangle$-module both by right multiplication and by inversion followed by left multiplication and in both cases B is a submodule of A. Set $T=\operatorname{Tr}_{1}\left(3, \mathrm{~J}^{2 \times 2}\right)<\operatorname{Tr}_{1}(6, \mathrm{~J})$. If $\mathrm{t}=\left(\mathrm{t}_{\mathrm{ij}}\right) \in \mathrm{T}$ (so $\mathrm{i}, \mathrm{j}=1,2,3$ and each $\mathrm{t}_{\mathrm{ij}}$ lies in $\left.\mathrm{J}^{2 \times 2}\right)$, then we can specify $\mathrm{t}$ simply by the triple $\left(\mathrm{t}_{21}, \mathrm{t}_{32}, \mathrm{t}_{31}\right)$. Let $\mathrm{d}=\operatorname{diag}\left(1_{2}, \mathrm{a}, 1_{2}\right) \in \operatorname{Tr}\left(3, \mathrm{~J}^{2 \times 2}\right)$. If $\mathrm{t}=(\lambda, \mu, \nu)$, then $\mathrm{d}^{-1} \mathrm{td}=\left(\mathrm{a}^{-1} \lambda, \mu \mathrm{a}, \nu\right)$. Set $\mathrm{G}=$ $\langle\mathrm{d}\rangle \mathrm{T} \leq \mathrm{GL}(6, \mathrm{~J})$.

Now $\mathrm{A}=\cup_{\mathrm{i}} 2^{-\mathrm{i}} \mathrm{B}$ and $2^{-i} \mathrm{~B} / 2^{-\mathrm{i}+1} \mathrm{~B}$ is centralised by $\langle\mathrm{a}\rangle$ on both sides (since $\mathrm{a} \equiv 1 \mathrm{mod}$ 2). Set $S=\operatorname{Tr}_{1}\left(3, \mathbb{Z}^{2 \times 2}\right)$ and $Z=\left(0,0, J^{2 \times 2}\right) \leq T$. Then $[Z, G]=\langle 1\rangle$ and $S$ is a finitely generated nilpotent subgroup of $\mathrm{T}$ with T/SZ is abelian, Chernikov and G-hypercentral. 
Let $x, y \in G$. Then $[x, y] \in T$. If $y \in T$, then $[x, 3 y]=1 \in S$. Suppose $x \in T$ and $y=t^{i}$, where $t \in T$, say $x=(\xi, \eta, \zeta)$ and $t=(\lambda, \mu, \nu)$. Then $[x, y]=\left[x, d^{i}\right][x, t]$ since $[x, t] \in Z$, which is central. Now $[\mathrm{x}, \mathrm{t}]=\eta \lambda-\mu \xi$ and

$$
\begin{aligned}
& {\left[x, d^{i}\right]=\left(\left(a^{-i}-1\right) \xi, \eta\left(a^{i}-1\right), \eta\left(1-a^{-i}\right) \xi\right), \text { so }} \\
& {[x, y]=\left(\left(a^{-i}-1\right) \xi, \eta\left(a^{i}-1\right), \eta\left(1-a^{-i}\right) \xi+\eta \lambda-\mu \xi\right) .}
\end{aligned}
$$

Thus $[\mathrm{x}, \mathrm{e}+1 \mathrm{y}] \in\left(\left(\mathrm{a}^{-\mathrm{i}}-1\right)^{\mathrm{e}+1} \xi, \eta\left(\mathrm{a}^{\mathrm{i}}-1\right)^{\mathrm{e}+1}, \pm \eta\left(\mathrm{a}^{\mathrm{i}}-1\right)^{2 \mathrm{e}+1}\langle\mathrm{a}\rangle \xi+\eta\left(\mathrm{a}^{\mathrm{i}}-1\right)^{\mathrm{e}} \lambda-\mu\left(\mathrm{a}^{-\mathrm{i}}-\right.\right.$ $1)^{e} \xi$ ) for all $e \geq 0$. Now 2 divides $a-1$. Therefore $[x, e+1 y] \in S$ for all large enough e (large here depending on $\mathrm{x}$ and $\mathrm{y}$ of course) and consequently $\mathrm{S}$ is both a sink and a knis for every $\mathrm{g}$ in $\mathrm{G}$. Therefore $\mathrm{G}$ is both left and right PF-Engel (in fact $\mathrm{G}$ is both left and right (finitely generated nilpotent)-Engel).

Let $\mathrm{N}$ be a normal finitely generated subgroup of $\mathrm{T}$. We claim that $\mathrm{N} \leq \mathrm{Z}$. If not consider any $x \in N Z$, say $x=(\xi, \eta, \nu)$. At least one of $\xi$ and $\eta$ is non-zero. Then

$$
\left[\left(0,2^{-\mathrm{h}} 1_{2}, 0\right), \mathrm{x}\right]=\left(0,0,2^{-\mathrm{h}} \xi\right) \in \mathrm{N} \cap \mathrm{Z} \text { and }\left[\mathrm{x},\left(2^{-\mathrm{h}} 1_{2}, 0,0\right)\right]=\left(0,0,2^{-\mathrm{h}} \eta\right) \in \mathrm{N} \cap \mathrm{Z}
$$

for all $h \geq 1$. But $N \cap Z$ is finitely generated and $\left\langle 2^{-h} \alpha: h \geq 1\right\rangle$ is not for any $\alpha \in J^{2 \times 2} \backslash\{0\}$. Thus we have the contradiction that $\xi=0=\eta$. Therefore $\mathrm{N} \leq \mathrm{Z}$.

Suppose $\mathrm{M}$ is a normal PF-Engel subgroup of $\mathrm{G}$ with $\mathrm{G} / \mathrm{M}$ hypercentral. We seek a contradiction. Now $\mathrm{M} \cap \mathrm{T}$ is a normal finitely generated subgroup of $\mathrm{T}$, so by the above $\mathrm{M} \cap \mathrm{T} \leq \mathrm{Z}$. Let $d^{i} x \in M$, where $x \in T$. Then $\left[G, d^{i} x\right] \leq M \cap T \leq Z$. In particular $\left[T, d^{i}\right] \leq\left[T, d^{i} x\right] Z \leq Z$, so $A\left(a^{i}-1\right)=\{0\}, a^{i}-1=0, i=0$ and $d^{i} x=x \in M \cap T \leq Z$. Consequently $M \leq Z$. It follows that $\mathrm{G} / \mathrm{Z}$ is hypercentral. This implies that a acts unipotently on $\mathrm{J}^{2 \times 2}$, say by right multiplication and consequently a $\in \operatorname{GL}(2, \mathbb{Z})$ is unipotent. But the eigenvalues of a are not 1 . This contradiction completes the proof that $\mathrm{G}$ is not $\mathbf{P F}$-by-hypercentral.

Finally $\mathrm{G}$ is connected; for $\mathrm{T}$, being unipotent in characteristic zero, is connected, so $\mathrm{T} \leq \mathrm{G}^{0}$. Also $\langle\mathrm{d}\rangle$ is connected since $\langle\mathrm{a}\rangle$ is, so $\langle\mathrm{d}\rangle \leq \mathrm{G}^{\mathrm{o}}$. Thus $\mathrm{G}=\langle\mathrm{d}\rangle \mathrm{T} \leq \mathrm{G}^{\mathrm{o}}$ and consequently $\mathrm{G}$ is connected.

\section{Compliance with ethical standards}

Conflict of interest The author states that there is no conflict of interest.

Open Access This article is licensed under a Creative Commons Attribution 4.0 International License, which permits use, sharing, adaptation, distribution and reproduction in any medium or format, as long as you give appropriate credit to the original author(s) and the source, provide a link to the Creative Commons licence, and indicate if changes were made. The images or other third party material in this article are included in the article's Creative Commons licence, unless indicated otherwise in a credit line to the material. If material is not included in the article's Creative Commons licence and your intended use is not permitted by statutory regulation or exceeds the permitted use, you will need to obtain permission directly from the copyright holder. To view a copy of this licence, visit http://creativecommons.org/licenses/by/4.0/.

\section{References}

1. Carter, R.W.: Simple Groups of Lie Type. Wiley, London (1972)

2. Casolo, C., Dardano, U., Rinauro, S.: Variants of theorems of Baer and Hall on finite-by-hypercentral groups. J. Algebra 452, 279-287 (2016)

3. de Falco, M., de Giovanni, F., Musella, C., Sysak, Y.P.: On the upper central series of infinite groups. Proc. Am. Math. Soc. 139, 385-389 (2011)

4. Khukhro, E.I., Shumyatsky, P.: Almost Engel compact groups. J. Algebra 500, 439-456 (2018) 
5. Khukhro, E.I., Shumyatsky, P.: Compact groups in which all elements are almost right Engel. Q. J. Math. 70, 879-893 (2019) (to appear)

6. Lennox, J.C., Robinson, D.J.S.: The Theory of Infinite Soluble Groups. Clarendon Press, Oxford (2004)

7. Robinson, D.J.S.: Finiteness Conditions and Generalized Soluble Groups (2 vols.). Springer, Berlin (1972)

8. Shirvani, M., Wehrfritz, B.A.F.: Skew Linear Groups. Cambridge University Press, Cambridge (1986) (re-issued 2008)

9. Shumyatsky, P.: Almost Engel linear groups. Monatsh. f. Math. 186, 711-719 (2018)

10. Shumyatsky, P.: Linear groups with almost right Engel elements. Proc. Edinb. Math. Soc. (2020). https:// doi.org/10.1017/s001309151800072x (to appear)

11. Thomas, S.: The classification of the simple periodic linear groups. Arch. Math. (Basel) 41, 103-116 (1983)

12. Wehrfritz, B.A.F.: Infinite Linear Groups. Springer, Berlin (1973)

13. Wehrfritz, B.A.F.: Variants of theorems of Schur, Baer and Hall. Ric. Mat. 67, 331-337 (2018)

14. Wehrfritz, B.A.F.: Weak Engel conditions on linear groups. Adv. Group Theory Appl. 7, 143-157 (2019)

15. Wehrfritz, B.A.F.: The structure of linear PF-Engel groups in characteristic zero. Boll. Un. Mat. Ital.

16. Wehrfritz, B.A.F.: Rank-Engel conditions on linear groups (preprint 2019)

Publisher's Note Springer Nature remains neutral with regard to jurisdictional claims in published maps and institutional affiliations. 